\title{
HOTAIRM1 regulates neuronal differentiation by modulating NEUROGENIN 2 and the downstream neurogenic cascade
}

\author{
Jessica Rea ${ }^{1}$, Valentina Menci ${ }^{1,6}$, Paolo Tollis ${ }^{1}$, Tiziana Santini', Alexandros Armaos ${ }^{3}$, Maria Giovanna Garone ${ }^{1}$

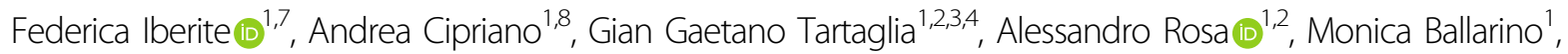 \\ Pietro Laneve ${ }^{5}$ and Elisa Caffarelli ${ }^{5}$
}

\begin{abstract}
Neuronal differentiation is a timely and spatially regulated process, relying on precisely orchestrated gene expression control. The sequential activation/repression of genes driving cell fate specification is achieved by complex regulatory networks, where transcription factors and noncoding RNAs work in a coordinated manner. Herein, we identify the long noncoding RNA HOTAIRM1 (HOXA Transcript Antisense RNA, Myeloid-Specific 1) as a new player in neuronal differentiation. We demonstrate that the neuronal-enriched HOTAIRM1 isoform epigenetically controls the expression of the proneural transcription factor NEUROGENIN 2 that is key to neuronal fate commitment and critical for brain development. We also show that HOTAIRM1 activity impacts on NEUROGENIN 2 downstream regulatory cascade, thus contributing to the achievement of proper neuronal differentiation timing. Finally, we identify the RNA-binding proteins HNRNPK and FUS as regulators of HOTAIRM1 biogenesis and metabolism. Our findings uncover a new regulatory layer underlying NEUROGENIN 2 transitory expression in neuronal differentiation and reveal a previously unidentified function for the neuronal-induced long noncoding RNA HOTAIRM1.
\end{abstract}

\section{Introduction}

Mammalian brain neurons derive from self-renewing neural stem cells (NSCs) whose differentiation occurs in two distinct steps: the initial production of neuronal precursors (NPs), already committed to a neuronal fate, and the following formation of differentiated cells that acquire and maintain their identity ${ }^{1}$.

Proneural genes (PGs) are key regulators of neurogenic differentiation. They encode transcription factors (TFs) of the basic helix-loop-helix (bHLH) class, mainly acting as transcriptional activators. In vertebrates, PGs are first

\footnotetext{
Correspondence: Pietro Laneve (pietro.laneve@cnr.it) or Elisa Caffarelli (elisa. caffarelli@cnr.it)

${ }^{1}$ Department of Biology and Biotechnology Charles Darwin, Sapienza University of Rome, Rome, Italy

${ }^{2}$ Center for Life Nano Science@Sapienza, Istituto Italiano di Tecnologia, Rome, Italy

Full list of author information is available at the end of the article

Edited by G. Melino
}

expressed in NSCs. However, their activity is carried out only later, when they reach high levels of expression, and gives rise to asymmetrically dividing NPs that are restricted to a neuronal fate. This is achieved through activation of neuronal-differentiation gene cascades, inhibition of glial cell fates and regulation of cell cycle ${ }^{2}$. The proneural TF NEUROGENIN 2 (NEUROG2) is a tightly regulated master gene in neuronal differentiation. In NSCs, it is controlled by the TF ETV5 ${ }^{3}$ and the bHLH repressors HES. In particular, the oscillatory and inverse correlated expression of HES genes and NEUROG2 impedes its neurogenic activity ${ }^{4,5}$, contributing to the maintenance of $\mathrm{NSCs}^{6-8}$. NEUROG2 is, instead, upregulated in NPs by the TFs PAX $6^{9}$ and SOX2, which acts in combination with the long noncoding RNA (lncRNA) RMST $^{10}$. NEUROG2 sustained and persistent expression instructs the neuronal differentiation programme by activating downstream targets that induce the expression

\section{(c) The Author(s) 2020}

(c) (i) Open Access This article is licensed under a Creative Commons Attribution 4.0 International License, which permits use, sharing, adaptation, distribution and reproduction in any medium or format, as long as you give appropriate credit to the original author(s) and the source, provide a link to the Creative Commons license, and indicate if changes were made. The images or other third party material in this article are included in the article's Creative Commons license, unless indicated otherwise in a credit line to the material. If material is not included in the article's Creative Commons license and your intended use is not permitted by statutory regulation or exceeds the permitted use, you will need to obtain permission directly from the copyright holder. To view a copy of this license, visit http://creativecommons.org/licenses/by/4.0/. 
of differentiation-related genes ${ }^{11}$ and the repression of the stemness factor PAX6 ${ }^{12}$.

Once the neuronal programme has been triggered, NEUROG2 expression must be turned off to allow the differentiation to proceed and to guarantee the maintenance of neuronal cell identity. We previously demonstrated that the Microprocessor complex, together with the RNA-binding protein TDP-43, is implicated in NEUROG2 gene silencing by inducing the degradation of its $\mathrm{mRNA}^{13}$. However, the mechanisms modulating NEUROG2 repression in differentiating neurons are still poorly characterised.

LncRNAs are powerful regulators of gene expres$\operatorname{sion}^{14,15}$. Notably, $40 \%$ of human-annotated lncRNAs are expressed in the brain where, through multiple mechanisms ${ }^{16}$, they impinge on every step of neurodevelopment, from differentiation to synaptogenesis ${ }^{14,17-19}$.

Herein, we assign a new function to the IncRNA HOTAIRM1 (HOXA Transcript Antisense RNA, Myeloid-Specific 1), a transcript previously implicated, as a cis-acting factor, in the regulation of HOXA gene clus$\operatorname{ter}^{20,21}$ and in myeloid maturation ${ }^{22}$. This study presents the first evidence of the involvement of HOTAIRM1 in neuronal differentiation. By exploiting different in vitro model systems, we demonstrate that, in the nucleus, the neuronal HOTAIRM1 isoform controls the transitory expression of NEUROG2, which impacts on the downstream neurogenic regulatory cascade.

\section{Materials and methods}

\section{Cell cultures and manipulations}

SH-SY5Y and NB4 cells were obtained from ATCC and DSMZ, respectively. Induced pluripotent stem cells (iPSCs) were derived and maintained as in ref. ${ }^{23}$. Details about cell culture, differentiation and transfection protocols are available in SI Materials and Methods. LNA GapmeRs and siRNAs are listed in Table S1.

\section{FISH and immunofluorescence}

SH-SY5Y cells and iPSC-derived human MNs were cultured on pre-coated glass coverslips and then fixed in $4 \%$ paraformaldehyde/PBS. FISH experiments were performed as described in ref. ${ }^{24}$ and in SI Materials and Methods.

\section{RNA extraction and analysis}

RNA expression analyses were performed through quantitative or semiquantitative real-time PCR (qRT-PCR or RT-PCR) on cDNA synthetised by Takara PrimeScript RT Reagent Kit (RR037A, Takara-bio).

\section{Cell fractionation}

Three-day RA-treated SH-SY5Y cells were fractionated according to the Ambion PARIS Kit (AM1921, Life Technologies). After RNA extraction, equal volumes of cytoplasmic or nuclear RNA were retro-transcribed and analysed by qRT-PCR. Normalisations were based on the total amount of RNA.

\section{Immunoblotting}

Whole-cell or nucleus/cytoplasm fractionated protein extracts were prepared from SH-SY5Y cells in RIPA buffer and processed as indicated in SI Materials and Methods.

\section{RNA immunoprecipitation (RIP) assay}

Nuclear RIP assay was carried out as in ref. ${ }^{13}$. Details and specific antibodies are reported in SI Materials and Methods.

\section{Chromatin immunoprecipitation (ChIP) assay}

Chromatin extracts were prepared from $1 \times 10^{6}$ proliferating or differentiating SH-SY5Y cells (eventually knocked-down for nHOTAIRM1) after cross-linking in $1 \%$ formaldehyde. Immunoprecipitation was performed using the MAGnify Chromatin Immunoprecipitation System kit (492024, Invitrogen). Details in SI Materials and Methods.

\section{Cross-linking immunoprecipitation (CLIP) assay}

Totally, $75 \times 10^{6} \mathrm{SH}-\mathrm{SY} 5 \mathrm{Y}$ cells were grown with RA for 6 days, UV cross-linked at $4000 \times 100 \mu \mathrm{j} / \mathrm{cm}^{2}$ and collected in NP-40 lysis buffer. Protocols for immunoprecipitation and RNA analysis are detailed in SI Materials and Methods.

\section{RNA antisense purification-mass spectrometry (RAP-MS)} assay

RAP-MS assay was performed on total cell extracts, as described in ref. ${ }^{25}$. Probe design, cell manipulation, experimental procedure and RNA and protein analyses are detailed in SI Materials and Methods.

\section{catRAPID analyses}

The catRAPID algorithm ${ }^{26}$ predicts the interaction potential of a protein and RNA pair. Interaction propensity is calculated from the primary structure alone ${ }^{26}$ and has been experimentally shown to estimate the binding affinity ${ }^{27,28}$. As demonstrated also in this work, catRAPID algorithm is able to separate interacting vs. non-interacting pairs with an area under the receiving operating characteristics (ROC) curve of 0.76 in $>500,000$ experimentally validated interactions ${ }^{29}$. Details of specific calculations for nHOTAIRM1 and U1 interactions are reported in SI Materials and Methods.

\section{Statistical analyses}

Histograms show the mean \pm SEM from 2 to 4 biological replicates. $N$ is indicated in Figure Legends. Errors 
were calculated from relative quantities and then opportunely propagated; statistical significance was determined by two-tailed paired Student's $t$ test. A $p$ value $(p)<0.05$ was considered as significant.

Analyses of variance were done using two-way ANOVA followed by post hoc Tukey's test (see SI Materials and Methods).

\section{Oligonucleotides}

Sequences of oligonucleotides are listed in Table S2.

\section{Results}

HOTAIRM1 and NEUROG2 expression are inversely correlated during neuronal differentiation

RNA-Seq analysis revealed that HOTAIRM1 is significantly upregulated in human neurons derived from iPSCs $^{30}$. By querying genotype-tissue expression (GTEx) Analysis Release V7 (dbGaP Accession phs000424.v7.p2) we found that, among thirteen brain tissues, HOTAIRM1 is exclusively expressed in the spinal cord (cervical c-1) (Fig. S1A). These lines of evidence suggested a possible role for HOTAIRM1 in the regulation of spinal neuron differentiation. To address this issue, we exploited human iPSCs induced to differentiate into ventral spinal cord lineages, which include about 30\% of motoneurons $(\mathrm{MNs})^{31}$ (Fig. S1B). In this context, we profiled the expression of HOTAIRM1 (Fig. 1a), whose genomic localisation is shown in Fig. S1C, together with that of the neuronal marker NEUROG2. HOTAIRM1 is induced at early stages (day 2), is stably accumulated until day 9 (NPs, marked by NEUROG2 (FIG. S1B)), reaches its maximum level in differentiating neurons (day 12), to strongly diminish in post-mitotic cells (day 16 , marked by CHAT and ISLET (Fig. S1B)). Notably, at the timewindow from day 9 to 12 an inverse correlation between HOTAIRM1 and NEUROG2 is evident and it was statistically corroborated by an overall significant two-way ANOVA analysis followed by Tukey's multiple comparisons test (alpha $=0.05)($ Dataset 1$)$. This result suggests a possible role for the IncRNA as a negative regulator of NEUROG2 expression in that time-window.

To investigate this potential function, we used the human neuroblastoma derived SH-SY5Y cells, considered to be $\mathrm{NPs}^{32}$. Upon retinoic acid (RA) treatment, they recapitulate the transition between NPs and differentiating neurons ${ }^{33}$, which occurs between day 9 and 12 of iPSC differentiation. RA administration produces a homogeneous population of mature neuron-like cells ${ }^{34,35}$, which express a variety of neuronal markers ${ }^{34,36}$. Figure $1 \mathrm{~b}$ shows a typical differentiation time course of SH-SY5Y cells treated with RA for 3 and 6 days. The levels of differentiation markers $V G F^{37}$ and $L A M C 1^{38}$ were gradually induced up to 6 days, whereas the proliferative marker $M Y C N^{39}$ dropped down already at 3 days. NEUROG2 and
HOTAIRM1 profiles showed their inversely correlated expression (Fig. 1c). The levels of HOTAIRM1 increased up to 6 days of RA treatment (about sixfold increase compared to control). Conversely, the levels of NEUROG2 were highest in undifferentiated SH-SY5Y cells according to their NP state ${ }^{33}$, drastically decreased at 3 days of RAtreatment and further reduced at later times (day 6). Accordingly, also the levels of NEUROG2 protein decreased (Fig. 1d). The expression profiles of HOTAIRM1 and NEUROG2 indicate that, both in differentiating iPSCs (Fig. 1a) and in RA-treated SH-SY5Y cells (Fig. 1c), the induction of HOTAIRM1 corresponds to a significant downregulation of NEUROG2.

\section{HOTAIRM1 is localised in both nuclear and cytoplasmic compartments of in vitro-derived neurons}

NCBI's Reference Sequence Database (RefSeq) in the UCSC Genome Browser (GRCh38/hg38) reports two HOTAIRM1 isoforms: the transcript variant 1 (HOTAIRM1_1; NR_038366.1), comprising three exons, and the transcript variant 2 (HOTAIRM1_2; NR_038367.1), lacking exon 2 (Fig. 2a). In RA-mediated myeloid differentiation, HOTAIRM1 was described as a unique transcript corresponding to HOTAIRM1_2 $2^{20}$, whereas a spliced isoform (HOTAIRM1_1) and an unspliced transcript were detected in an axial development system ${ }^{21}$.

By semi-qRT-PCR, we analysed HOTAIRM1 variants both in differentiating iPS and SH-SY5Y cells and, as control, in NB4 promyelocytic leukaemia cells induced to myeloid differentiation ${ }^{20}$. Figure $2 \mathrm{~b}$ shows that only HOTAIRM1_1 was detected in differentiating iPSCs (left panel) and that it represents the major isoform in RAtreated SH-SY5Y cells (middle panel). HOTAIRM1_2 was, instead, the only transcript observed in RA-treated NB4 cells (right panel and ref. ${ }^{20}$ ). These findings indicate that, while HOTAIRM1_2 is the myelopoietic-enriched transcript, HOTAIRM1_1 represents the neuronal-enriched isoform. We addressed the following molecular, functional and mechanistic studies on this isoform, referred hereafter as nHOTAIRM1 (neuronal HOTAIRM1).

As HOTAIRM1_2 $2^{20}$, nHOTAIRM1 is a polyadenylated transcript (Fig. 2c). Its subcellular localisation was explored through both biochemical fractionation and RNA fluorescence in situ hybridisation (FISH) analyses. Subcellular fractionations indicated that nHOTAIRM1 was present both in the nucleus and cytoplasm of proliferating (day 0 ) and differentiating (day 6) SH-SY5Y cells, the cytoplasmic component being more abundant (Fig. 2d). RA-mediated upregulation of nHOTAIRM1 was comparable in both compartments (about sixfold increase; Fig. 2e).

FISH analysis was performed in fluorescence-activated cell sorting (FACS)-purified MNs derived from iPSCs upon 12 days of differentiation (Fig. 2f) and, as control, in RNase-treated MNs (Fig. S2). According to biochemical 


\section{A D HOTAIRM1 $\square$ NEUROG2}
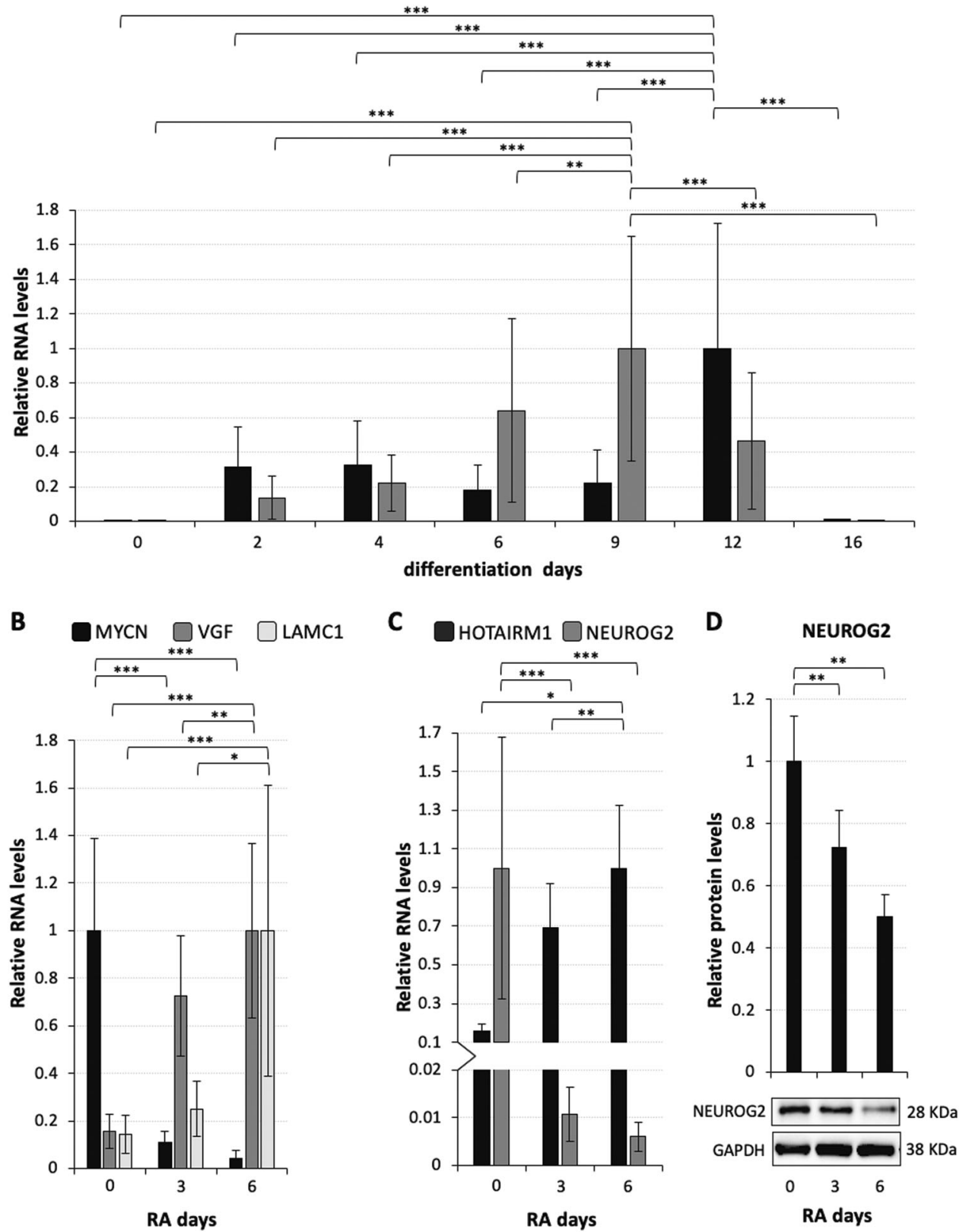

Fig. 1 HOTAIRM1 and NEUROG2 expression are inversely correlated during neuronal differentiation. a qRT-PCR analysis of HOTAIRM1 and NEUROG2 expression along differentiation of iPSCs into ventral spinal cord lineages. Differentiation days are reported on the $x$-axis. Expression levels are relative to ATP5O and expression peaks are set as $1 . N=1,2$ or 3 , depending on the sample. ${ }^{* *} P \leq 0.0021,{ }^{* * *} P \leq 0.0002$. $\mathbf{b}$ qRT-PCR analysis of mRNAs coding for MYCN (proliferative marker), VGF (neuropeptide precursor) and LAMC1 (neurite-promoting factor) along neuronal differentiation of SH-SY5Y cells. Days of RA treatment are reported on the $x$-axis. Expression levels are relative to GAPDH, expression peaks are set as $1 . N=3$, ${ }^{*} P \leq 0.05$, ${ }^{* *} P \leq 0.01,{ }^{* *} P \leq 0.001$. c qRT-PCR analysis of HOTAIRM1 and NEUROG2 expression along neuronal differentiation of SH-SY5Y cells. Broken axishistogram allows to appreciate low values. Details as in (b). $N=2$ or 3 , depending on the target. ${ }^{*} P \leq 0.05,{ }^{* *} P \leq 0.01$, ${ }^{* * *} P \leq 0.001$. d Immunoblot analysis of NEUROG2 along neuronal differentiation of SH-SY5Y cells. Days of RA treatment are reported below each lane. In the histogram, NEUROG2 levels are relative to GAPDH. $N=3,{ }^{* *} P \leq 0.01$.

data, this analysis confirmed the double localisation of the lncRNA in post-mitotic neurons (Fig. 2f, panels 1 and 2). Notably, cytoplasmic nHOTAIRM1 was localised both in the soma and neurites of MNs, suggesting it might participate in local RNA regulation (Fig. 2f, panel 3). Altogether, these results indicate that nHOTAIRM1 has a dual 


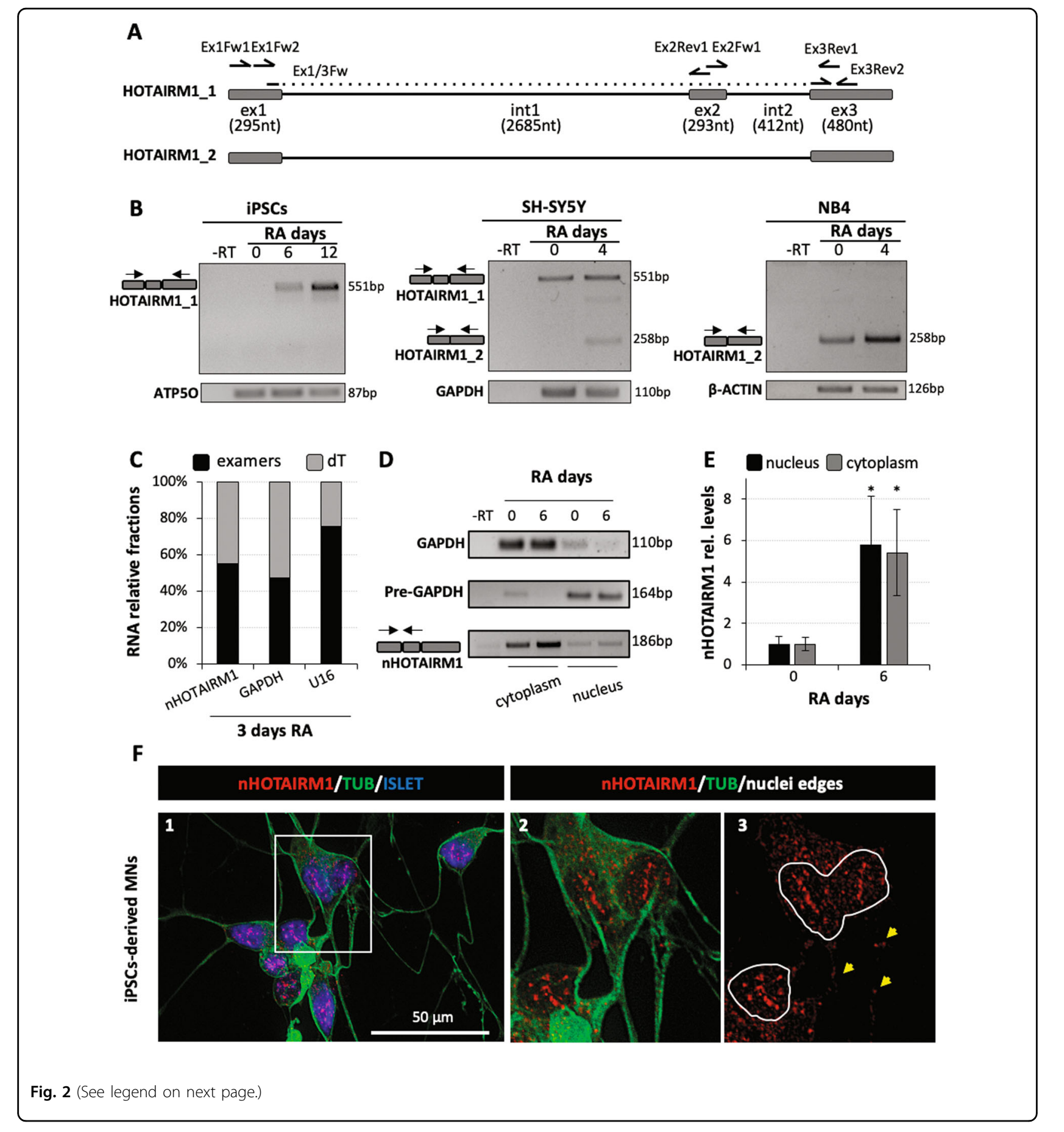

cellular localisation and, in principle, it might exert different functions in different cell compartments.

\section{Nuclear nHOTAIRM1 regulates NEUROG2 and the} downstream neurogenic cascade

Based on the inverse correlation between nHOTAIRM1 and NEUROG2 expression (Fig. 1a, c) we ascertained, by a loss-of-function approach, whether nHOTAIRM1 may act as a negative regulator of NEUROG2 during neuronal differentiation. To differentially knockdown nuclear and cytoplasmic nHOTAIRM1 species, we designed two sets of antisense oligonucleotides: LNA GapmeRs, more effective for nuclear targets, and siRNAs, that function preferentially in the cytoplasm ${ }^{40,41}$ (Fig. 3a).

RA-treated SH-SY5Y cells were transfected with siRNAs or LNA GapmeRs, and RNA purified from nuclear 


\begin{abstract}
(see figure on previous page)
Fig. 2 Molecular characterisation of HOTAIRM1. a Schematic representation of HOTAIRM1 transcript variants (HOTAIRM1_1 and HOTAIRM1_2) according to UCSC Genome browser. Exons (ex) are indicated as boxes, introns (int) as a continuous line. Lengths (nucleotides, nt) are reported between brackets. Oligonucleotides used for semi-qRT-PCR and qRT-PCR amplifications are indicated. b Semi-qRT-PCR analysis of HOTAIRM1 isoforms (described in panel (a)) in iPS (left panel), SH-SY5Y (middle panel) and NB4 (right panel) differentiating cells. Differentiation days are indicated above each lane. -RT samples were used as negative controls and ATP5O, GAPDH or $\beta$-ACTIN as internal standards. Amplifications were performed through primers Ex1FW1 and Ex3Rev1, indicated in panel (a). Expected amplicon sizes are indicated on the right. c qRT-PCR analysis of HOTAIRM1_1 (nHOTAIRM1) polyadenylated/un-polyadenylated fractions (expressed as \% of total transcript levels) in 3-day RA-treated SH-SY5Y cells. Reverse transcription was performed by random examers (examers) or dT oligonucleotides (dT). GAPDH and U16 snRNA were used as representative polyadenylated or un-polyadenylated species, respectively. Amplifications were performed through primers Ex2Fw1 and Ex3Rev1. N=1. d Semi-gRTPCR analysis of nHOTAIRM1 subcellular localisation in nuclear and cytoplasmic fractions of undifferentiated (day 0) and 6-day RA-treated (day 6) SHSY5Y cells. Fractionation efficiency was assessed by GAPDH and pre-GAPDH analysis in the nucleus and cytoplasm, respectively. -RT samples were used as negative controls. $\mathrm{nHOTAIRM} 1$ amplifications were performed through primers Ex1Fw2 and Ex2Rev1. Expected amplicon sizes are indicated on the right. e qRT-PCR analysis of nHOTAIRM1 in the nucleus and the cytoplasm of differentiating SH-SY5Y cells. Expression at day 0 was set as 1. Normalisation was performed on total RNA. $N=3,{ }^{*} P \leq 0.05$. f nHOTAIRM1 localisation in FACS-purified MNs. Panel 1: RNA FISH analysis combined with immunofluorescence in MNs purified by FACS from differentiated human iPSCs; nHOTAIRM1, TUBULIN III (TUB) and ISLET 1/2 (ISLET) are stained in red, green and blue, respectively. Panel 2: digital magnification of the square insert of panel 1, without DAPI staining. Panel 3: the same picture as in panel 2 with nuclei edges; yellow arrowheads point to nHOTAIRM1 FISH signals in the neurites.
\end{abstract}

and cytoplasmic fractions (Figs. S3A, B) was analysed by qRT-PCR. LNA GapmeR1 caused a 60\% reduction of both cytoplasmic and nuclear nHOTAIRM1 (Fig. 3b), whereas siRNAs exclusively knocked-down cytoplasmic nHOTAIRM1 (by about 60\%) (Fig. 3c). Therefore, the parallel use of the two sets of antisense oligonucleotides allowed us to discriminate between nuclear and cytoplasmic nHOTAIRM1 functions.

We evaluated NEUROG2 levels in 3-day RA-treated cells upon siRNA- or GapmeR-mediated nHOTAIRM1 silencing. No any modulation of NEUROG2 was observed when the expression of cytoplasmic nHOTAIRM1 was silenced by siRNAs targeting two different lncRNA regions (Figs. 3d, S4A). Differently, an about twofold increase of NEUROG2 mRNA levels was achieved upon $80 \%$ reduction of nHOTAIRM1 by different LNA GapmeRs directed against the neuronal isoform (Figs. 3e, S4B). Pursuant to NEUROG2 mRNA upregulation, a sixfold increase of NEUROG2 protein was detected (Fig. 3f). These data demonstrated that only the nuclear nHOTAIRM1 negatively controls NEUROG2 expression. As a specificity-control, we tested whether HOTAIRM1 may functionally interact with $M Y C N$, which, similarly to NEUROG2, showed an inverse expression profile with the lncRNA during differentiation (Fig. 1b). Interestingly, we found that HOTAIRM1 GapmeR-mediated knockdown did not impact $M Y C N$ expression in differentiating $\mathrm{SH}-$ SY5Y cells (Fig. S4C).

To realise the full implications of the aberrant increase of NEUROG2 protein, we evaluated the levels of two downstream targets in the neurogenic cascade, the proneural factors $N E U R O D$ and ASCL1. NEUROG2 activates $N E U R O D^{11}$ and represses $A S C L 1^{42}$ expression during differentiation. LNA GapmeR-mediated knockdown of nHOTAIRM1 (GapmeR1, Fig. 3e) caused an increase of
NEUROD mRNA levels by $~ 50 \%$ and a decrease of ASCL1 mRNA by $\sim 80 \%$ (Fig. 3g). No any variation of these NEUROG2 targets was observed when nHOTAIRM1 silencing was performed with siRNAs (siRNA1, Fig. 3h). These results, confirmed by the use of additional siRNAs (siRNA2, Fig. S4D) and LNA GapmeRs (GapmeR2, Fig. S4E), entail that nuclear nHOTAIRM1 regulates NEUROG2 expression and its downstream neurogenic cascade during in vitro neuronal differentiation.

\section{Nuclear nHOTAIRM1 is required for the epigenetic regulation of NEUROG2 expression}

Nuclear lncRNAs often function as molecular scaffolds mediating epigenetic modifications in cis and/or in trans ${ }^{16}$. It has been shown that HOTAIRM1 recruits both polycomb repressive complex 2 (PRC2) and trithorax complex during axial development ${ }^{21}$. Based on these observations, we asked whether nHOTAIRM1 affected the epigenetic status of NEUROG2 promoter. First, we examined whether NEUROG2 expression was epigenetically silenced during neuronal differentiation. To this aim, we evaluated the activity of PRC2, which promotes the epigenetic silencing by $\mathrm{H} 3 \mathrm{~K} 27$ trimethylation ${ }^{43-46}$, on NEUROG 2 promoter ${ }^{4}$ in undifferentiated vs. RA-treated SH-SY5Y cells. Chromatin immunoprecipitation (ChIP) assay, performed on two selected promoter regions (Fig. S5A), revealed a twofold increase of H3K27me3 repressive mark at 3 days of differentiation (Fig. 4a), which correlates with the observed drastic reduction of NEUROG2 expression (Fig. 1c). Increased H3K27me3 enrichment was not observed on the promoter of the HPRT1 control gene (Fig. S5B, C).

To test the involvement of nHOTAIRM1 in such epigenetic silencing, we performed ChIP analysis in RA- 
A

1. ECTOPIC EXPRESSION:

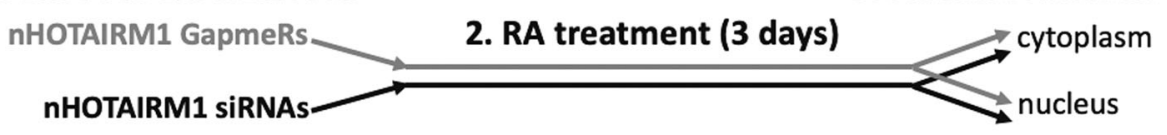

B
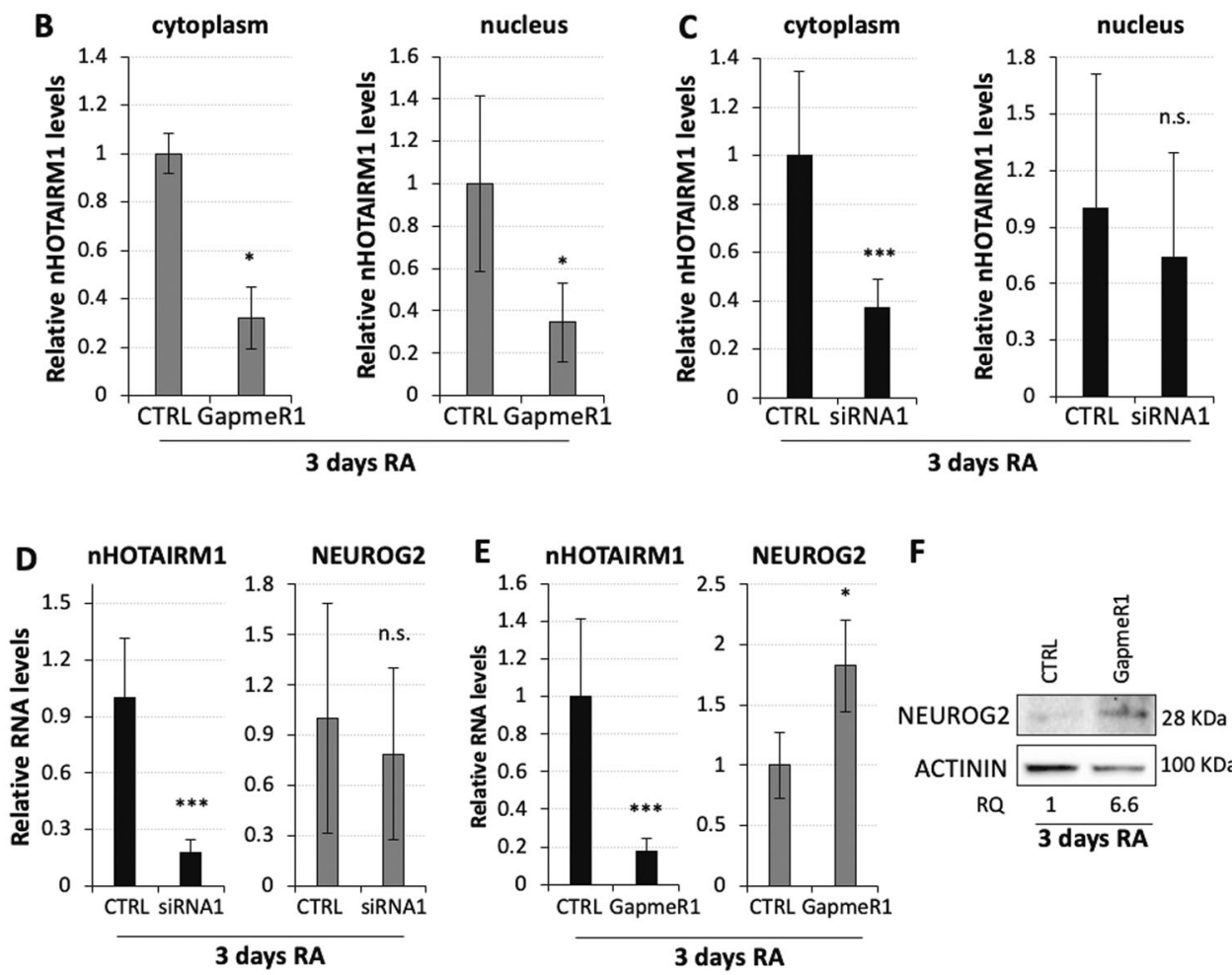

$F$
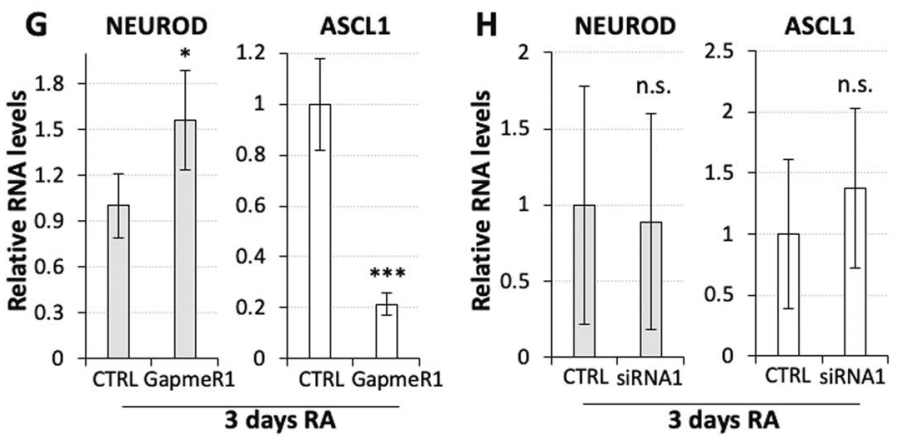

Fig. 3 (See legend on next page.)

treated cells upon nHOTAIRM1 knockdown by LNA GapmeRs. Depletion of nHOTAIRM1 by $80 \%$ (Fig. 3e) caused a significant reduction of H3K27me3 deposition on both regions of NEUROG2 promoter (Fig. 4b), while it did not affect the control gene (Fig. S5D). This result demonstrates that nHOTAIRM1 is implicated in the control of the epigenetic status of NEUROG2 during neuronal differentiation, by favouring H3K27 trimethylation.

To corroborate this evidence, we monitored the ability of the lncRNA to associate with PRC2 by RNA immunoprecipitation assay (RIP assay) and by RNA FISH combined with immunofluorescence analysis (RNA FISH/ IF). Immunoprecipitation of the PRC2 component 
(see figure on previous page)

Fig. 3 nHOTAIRM1 loss-of-function affects the expression of NEUROG2 and its target genes. a Schematic representation of the experimental design. nHOTAIRM1 LNA GapmeRs or siRNAs are ectopically expressed in differentiating SH-SY5Y cells, then separated into nuclear and cytoplasmic fractions. $\mathbf{b}$ qRT-PCR analyses of nHOTAIRM1 expression in the cytoplasm (left panel) and the nucleus (right panel) of 3-day RA treated SH-SY5Y cells, transfected with LNA GapmeRs (GapmeR1). Expression was set as 1 in scrambled-transfected cells (CTRL). Normalisation was performed on total RNA. $N=3$, ${ }^{*} P \leq 0.05$. c qRT-PCR analysis of nHOTAIRM1 expression in the cytoplasm (left panel) and the nucleus (right panel) of 3-day RA treated SH-SY5Y cells, transfected with siRNAs (siRNA1). Details as in (b). $N=4$, ${ }^{* *} P \leq 0.001$. d qRT-PCR analyses of nHOTAIRM1 (left histogram) and NEUROG2 (right histogram) expression upon siRNA-mediated nHOTAIRM1 knockdown (siRNA1) in 3-day RA treated SH-SY5Y cells. Scrambled siRNAs were used as negative control (CTRL) and set as 1. Data are relative to GAPDH. N $=3$, ${ }^{* * *} P \leq 0.010$. e qRT-PCR analyses of nHOTAIRM1 (left histogram) and NEUROG2 (right histogram) expression upon GapmeR-mediated nHOTAIRM1 knock-down (GapmeR1) in 3-day RA treated SH-SY5Y cells. Scrambled GapmeRs were used as negative control (CTRL) and set as 1 . Details as in (d). $N=3$ or 4 , depending on the target. ${ }^{*} P \leq 0.05$, ${ }^{* *} P \leq 0.001$. f Immunoblot analysis of NEUROG2 protein levels upon GapmeR1-mediated nHOTAIRM1 knockdown in 3-day RA treated SH-SY5Y cells. Scrambled GapmeRs were used as negative control (CTRL) and ACTININ as internal standard. Relative quantity (RQ) was expressed with respect to control cells set as 1 and reported below each lane. $N=1$. $\mathbf{g}$ qRT-PCR analyses of NEUROD (left histogram) and ASCL1 (right histogram) expression upon GapmeR1-mediated nHOTAIRM1 knockdown in 3-day RA treated SH-SY5Y cells. Details as in (d). $N=4,{ }^{*} P \leq 0.05,{ }^{* *} P \leq 0.001$. $\mathbf{h}$ qRT-PCR analyses of $N E U R O D$ (left histogram) and ASCL1 (right histogram) expression upon siRNA1-mediated nHOTAIRM1 knockdown in 3-day RA treated SH-SY5Y cells. Details as in (d). $N=2$.

SUZ12, performed in nuclear extracts from differentiating SH-SY5Y cells (Fig. 4c, upper panel), revealed the interaction between SUZ12 and nHOTAIRM1 (Fig. 4c, lower panel). In line with this result, a combined FISH/IF approach highlighted the partial overlap between nHOTAIRM1 RNA and PRC2 signals (Fig. 4d, e, magnified in Fig. 4f, panel 1). In particular, $\sim 25 \%$ of the nHOTAIRM1 signals showed spatial proximity with the PRC2 catalytic component EZH2 at the same focal planes, indicating 3D-colocalization of the signals, as shown by isosurface rendering (Fig. 4f, panel 2) and fluorescence plot (Fig. 4g).

Supporting the role of nHOTAIRM1 as a direct interactor of NEUROG2 locus, RNA/DNA FISH revealed the spatial proximity between the lncRNA and NEUROG2 genomic region in differentiating SH-SY5Y cells (Fig. 4h). As shown in the histogram aside, $\sim 35 \%$ of spots corresponding to NEUROG2 loci (green signals) are in paired or overlapped configuration to nHOTAIRM1 spots (red signals).

We conclude that nHOTAIRM1 is functionally required for recruiting the epigenetic machinery that catalyses H3K27me3 modification on NEUROG2 locus, during neuronal differentiation.

\section{Identification of nHOTAIRM1 protein interactors}

RNAs are co-transcriptionally assembled into functional ribonucleoprotein particles ${ }^{47}$ whose protein moiety is informative on both RNA metabolism and activity. To identify nHOTAIRM1 direct protein partners in living cells, we exploited RNA antisense purification (Fig. 5a) coupled with mass spectrometry (RAP-MS) analysis ${ }^{25}$. The experiment was performed in differentiating spinal MNs-expressing high levels of nHOTAIRM1 (Fig. S6A) -derived from human iPSCs through a fast method ${ }^{48,49}$. To assess whether RAP worked properly, it was applied in parallel to U1 snRNA (Fig. S6B), whose protein interactors are well-known ${ }^{50}$. Indeed, U1 RAP-MS analysis revealed strong enrichment of U1snRNP specific components, as SNRNP70, SNRPA and SNRPC and the Sm proteins SNRPB, SNRPD1, SNRPD2, SNRPD3, SNRPE and SNRPGP15 (Dataset 2).

To produce a list of nHOTAIRM1 high-confidence protein interactors, we combined the experimentally identified hits with the in silico calculations generated through the catRAPID algorithm (Materials and methods) $)^{51,52}$ that eliminate any experimental bias between the sample and the control. Analysing about 3500 potential protein-RNA interactions, we found strong agreement between catRAPID predictions and RAP-MS experiments, with an area under the ROC curve of 0.75 . In Fig. $5 \mathrm{~b}$, we report the proteins with strong catRAPID interaction propensities (scores $>2$ ) and detected in RAPMS with an normalised spectrum abundance factor (NSAF) score $>0.01$ (Dataset 2). nHOTAIRM1 direct interactors include SR-splicing factors, as SFPQ ${ }^{53}$, SRSF1, SRSF3, SRSF7 ${ }^{54}$ and heterogeneous nuclear ribonucleoproteins (HNRNPs) ${ }^{55}$ as HNRNPA1, HNRNPH1, HNRNPH2, HNRNPF, HNRNPK and FUS ${ }^{56}$. Directly comparing catRAPID interaction propensities for nHOTAIRM1 and U1, we identified HNRNPK and FUS as the hits with the largest binding preference for nHOTAIRM1 (Dataset 2, Column I). Thus, following the experimental and computational prioritisation of HNRNPK and FUS, we decided to further investigate their roles in nHOTAIRM1 function and metabolism.

\section{The RNA-binding proteins HNRNPK and FUS control nHOTAIRM1 levels}

The HNRNPK protein, whose levels are downregulated during in vitro neuronal differentiation (Fig. S7A and ref. ${ }^{57}$ ), is involved in several cellular pathways such as 

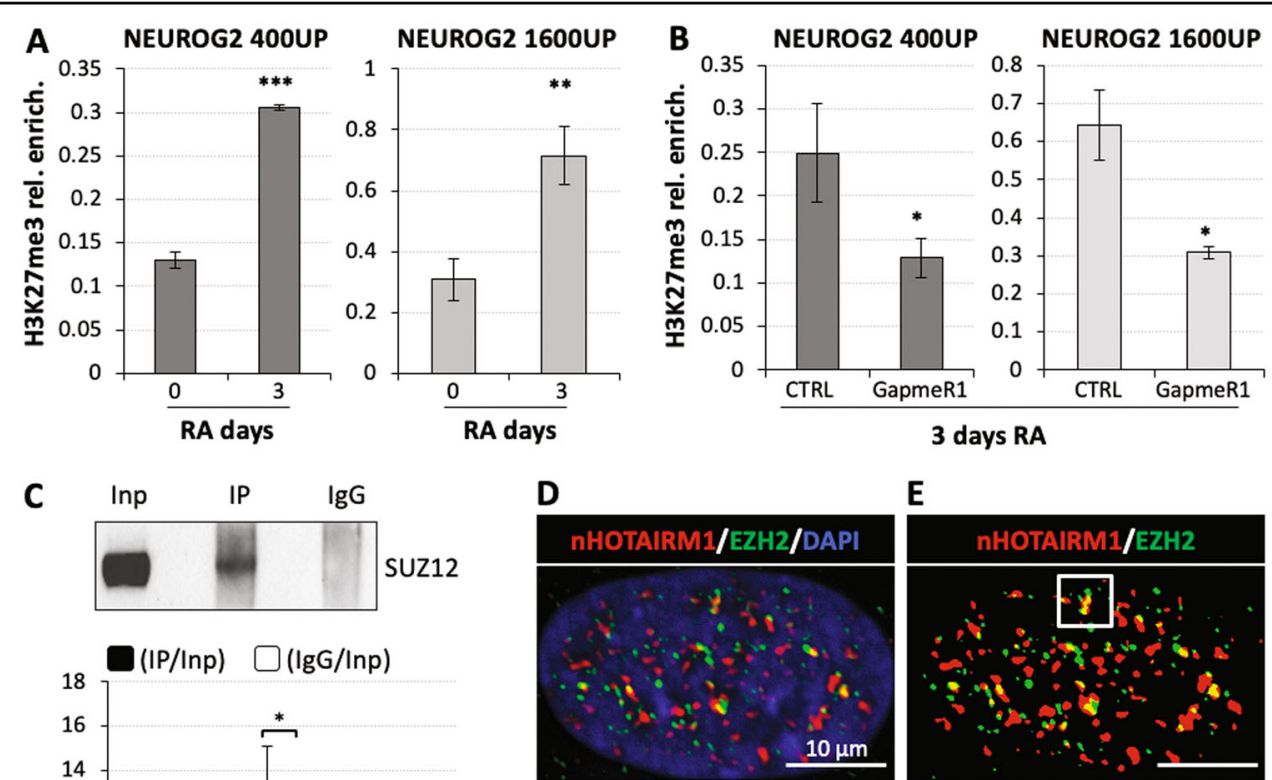

E
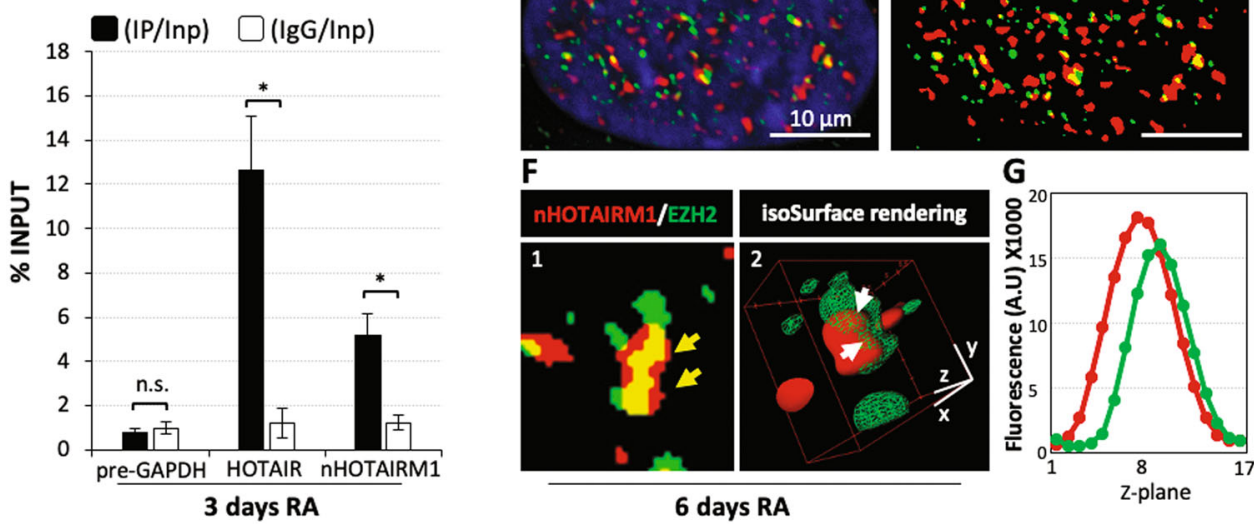

H
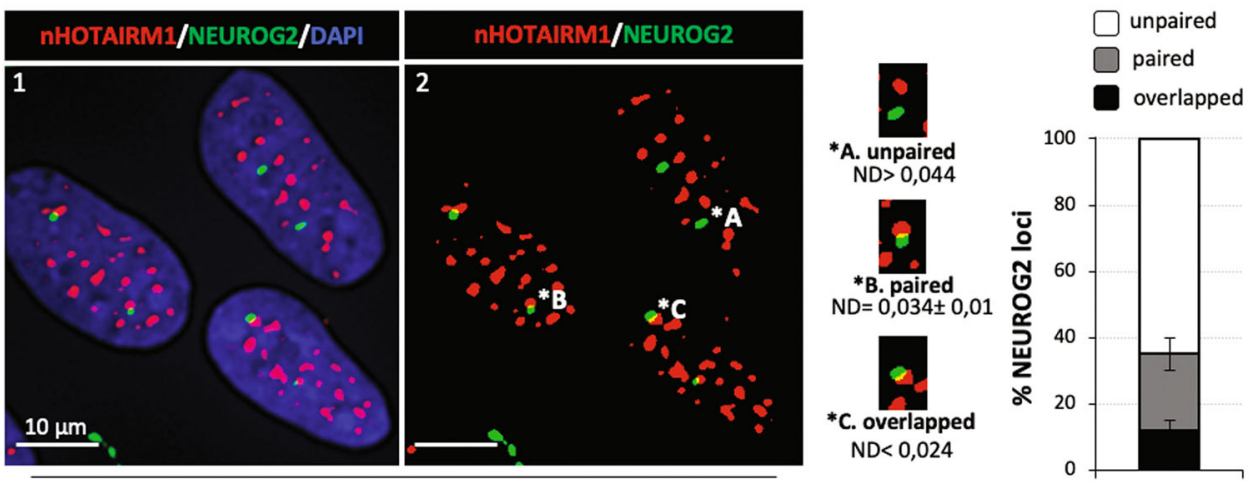

6 days RA

Fig. 4 (See legend on next page.)

transcription, splicing and translation ${ }^{58}$. To understand the significance of its interaction with nHOTAIRM1, we knocked down HNRNPK expression in differentiating SH-SY5Y cells and evaluated the lncRNA levels. A decrease of $\sim 30 \%$ of HNRNPK mRNA (Fig. S7B) and protein (Fig. 5c) levels caused an increase of both mature (about $80 \%$, Fig. 5d) and primary (about 40\%, Fig. 5e)
nHOTAIRM1 transcripts. In line with HNRNPK activity as a transcriptional repressor ${ }^{58}$ this may be explained, at least in part, by lncRNA transcriptional activation. Remarkably, a significant accumulation of the myeloidenriched variant HOTAIRM1_2, which is normally absent or expressed at low levels in neuronal systems (Fig. 2b), was also observed upon HNRNPK depletion (about 300\% 
(see figure on previous page)

Fig. 4 nHOTAIRM1 epigenetically regulates NEUROG2 through PRC2. a H3K27me3 occupancy on regions 400 bp (400UP, left panel) or 1600 bp (1600UP, right panel) upstream of NEUROG2 TSS (see Fig. S5A) in SH-SY5Y differentiating cells (day 3), compared to untreated cells (day 0). An intergenic region (2000 bp upstream of the HPRT1 TSS; see Fig. S5B) was used to normalise the two conditions. Enrichments are expressed as percentage relative to Input. $N=4,{ }^{* *} P \leq 0.01,{ }^{* * *} P \leq 0.001$. b H3K27me3 occupancy on NEUROG2 upstream regions upon nHOTAIRM1 knockdown (GapmeR1) compared to control-transfected cells (CTRL), in 3-day RA-treated SH-SY5Y cells. Details as in (a). ${ }^{*} P \leq 0.05$. c SUZ12-mediated RNA immunoprecipitation assay in 3-day RA-treated SH-SY5Y cells. Upper panel: immunoblot analysis of SUZ12 in Input (Inp), immunoprecipitated (IP) and $\mathrm{lgG}(\mathrm{lgG})$ protein fractions. Lower panel: RNA enrichment over Input, in IP and IgG protein fractions. Data are expressed as percentage of Input. PreGAPDH and HOTAIR enrichments were used as negative and positive controls, respectively. $N=3,{ }^{*} P \leq 0.05$. d Combined RNA FISH and IF analyses showing the spatial correlation between nHOTAIRM1 and EZH2 in 6-day RA-treated SH-SY5Y cells: representative fluorescence image (Z-projection of confocal stacks) indicating the localisation of nHOTAIRM1 RNA (red signals) and EZH2 protein (green signals) in the nucleus (DAPI, blue staining). e RNA FISH/IF staining presented in panel (d) converted to a binary image, to highlight the overlapping signals (yellow). $\mathbf{f}$ Magnification of the square insert in (e) showing the colocalized regions (yellow arrows) in a 2D representation (panel 1) and in a 3D volume rendering (isosurface) (panel 2). In the latter panel, white arrows show the local points where nHOTAIRM1 RNA and PRC2 signals are overlapped. $\mathbf{g}$ Plot of Z-stack intensity distribution for colocalized nHOTAIRM1/EZH2 signals reported in panel (f). The curves show the fluorescence intensity profile in each channel (A.U. = arbitrary units) along Z-confocal planes $(Z$-step $=200 \mathrm{~nm})$. The correspondence of the distributions indicates the 3D spatial correlation of the signals. The 3Dcolocalization between nHOTAIRM1 and EZH2 was detected in $26.32 \% \pm 1.5$ of nHOTAIRM1 RNA FISH signals analysed in 39 nuclei (mean \pm SEM, $N=$ 3). h RNA/DNA FISH for nHOTAIRM1 RNA (red signals) and NEUROG2 locus (green signals) in 6-day RA treated SH-SY5Y (panel 1). Signal conversion into binary image is shown in panel 2: magnifications of three representative conditions (unpaired, paired or overlapped spots) are reported aside. Histogram on the right shows a quantitative analysis, in one representative experiment, of NEUROG2 loci unpaired, paired or overlapped to nHOTAIRM1 spots, expressed as the mean \pm SD percentage. Scale bar $=10 \mu \mathrm{m}$.

increase, Fig. 5f). Altogether, these results suggest that HNRNPK participates in the control of nHOTAIRM1 metabolism, at both the transcriptional and splicing levels.

Next, we asked whether the HNRNPK-mediated nHOTAIRM1 gain-of-function (Fig. 5d) may impinge on NEUROG2 pathway. We found that HNRNPK silencing in differentiating SH-SY5Y cells caused a 30\% decrease of NEUROG2 mRNA (Fig. 5g) and a $50 \%$ reduction of NEUROG2 protein (Fig. 5h), which resulted in the alteration of NEUROD and ASCL1 expression (Fig. 5g). This is consistent with an increased level of nHOTAIRM1 in the nucleus as verified by cell fractionation (Fig. 5i). Notably, the effect of nHOTAIRM1 gain-of-function is complementary to the one obtained through its silencing (Fig. 3e, g), leading to the conclusion that both loss- and gain-of-function of nuclear nHOTAIRM1 impinge on NEUROG2 cascade.

The second ranked nHOTAIRM1 interactor is FUS, an RNA-binding protein downregulated during neuronal differentiation (Fig. S8A and ref. ${ }^{59}$ ), which plays a relevant role in brain development and neurodegeneration ${ }^{60-62}$. FUS knockdown experiments in differentiating SH-SY5Y cells produced an $80 \%$ reduction of FUS mRNA and protein (Figs. S8B, Fig. 6a). This caused a decrease of nHOTAIRM1 levels by $\sim 40 \%$ (Fig. 6b). Nevertheless, FUS knockdown did not cause any alteration of NEUROG2, NEUROD and ASCL1 mRNAs (Fig. 6c). This is in line with the finding that FUS silencing affected only the cytoplasmic nHOTAIRM1 transcript (Fig. 6d). Accordingly, CLIP assay revealed FUS/nHOTAIRM1 interaction in the cytoplasm (Fig. 6e). These data unveil FUS as a direct regulator of nHOTAIRM1 abundance in the cytoplasm and confirm that nHOTAIRM1 cytoplasmic transcript does not participate in the control of the NEUROG2-mediated pathway.

\section{Discussion}

Fine regulation of NEUROG2 during differentiation is crucial for the entire neurogenesis. Even subtle alterations of its expression are detrimental for brain development and organisation ${ }^{63}$. High levels of NEUROG2 in NPs are required to instruct neuronal differentiation, by inducing neurogenic gene cascades and by repressing genes responsible for cell proliferation. Instead, low levels of the protein are essential in differentiating neurons to allow the temporal progression of neurogenesis and to maintain their differentiated state. Therefore, NEUROG2 abundance is an important determinant controlled by multiple regulatory mechanisms that, acting at proper differentiation times, secure the neuronal cell fate. So far, both transcriptional $^{5,9}$ and post-transcriptional mechanisms ${ }^{13,64}$ have been described for NEUROG2 repression during neuronal differentiation (Fig. 7). Here, we discover a novel epigenetic layer of regulation for NEUROG2 and highlight the contribution of the IncRNA nHOTAIRM1 to this control.

To perform molecular, functional, and mechanistic studies, we exploited two neuronal differentiation models that recapitulate the neuronal differentiation in vitro, the iPS and the SH-SY5Y cells. Using these systems we: (i) profiled the expression of NEUROG2 and nHOTAIRM1 during neuronal differentiation, (ii) underscored their inverse correlation in the transition between NPs and differentiating neurons, (iii) characterised the neuronal isoform of HOTAIRM1, unveiling nuclear and cytoplasmic transcripts and (iv) demonstrated that the nuclear lncRNA is required for NEUROG2 repression at the proper differentiation time. Importantly, perturbations of the lncRNA levels impinge on at least two direct targets of NEUROG2, namely NEUROD, whose activation 


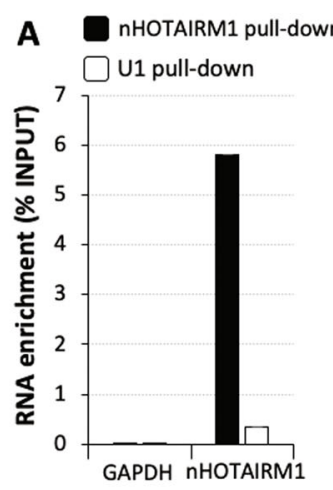

B

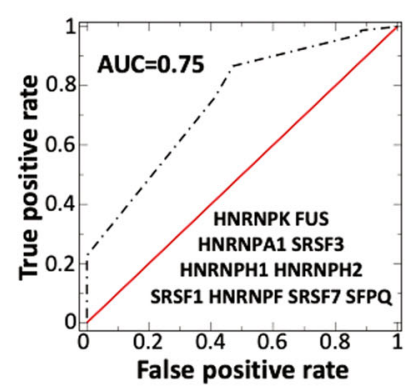

E HOTAIRM1 precursor
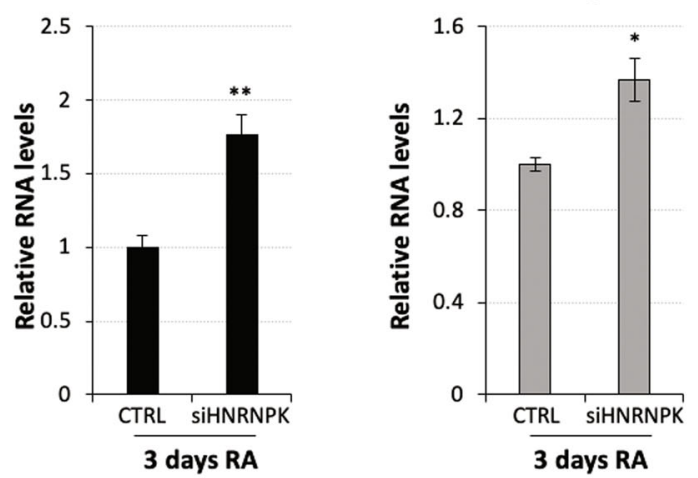

G $\square$ neUrog $\square$ neurod $\square$ ascl1

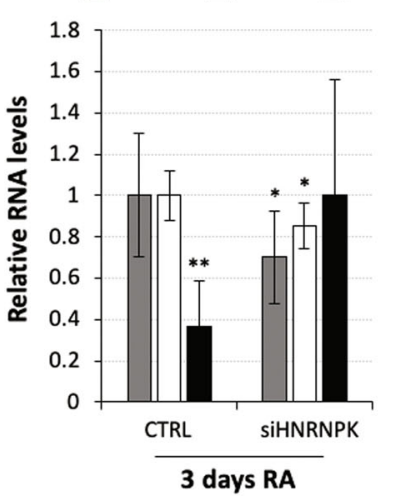

C

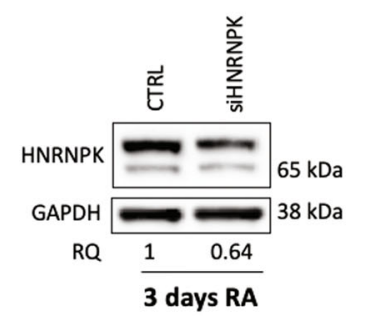

F HOTAIRM1_2
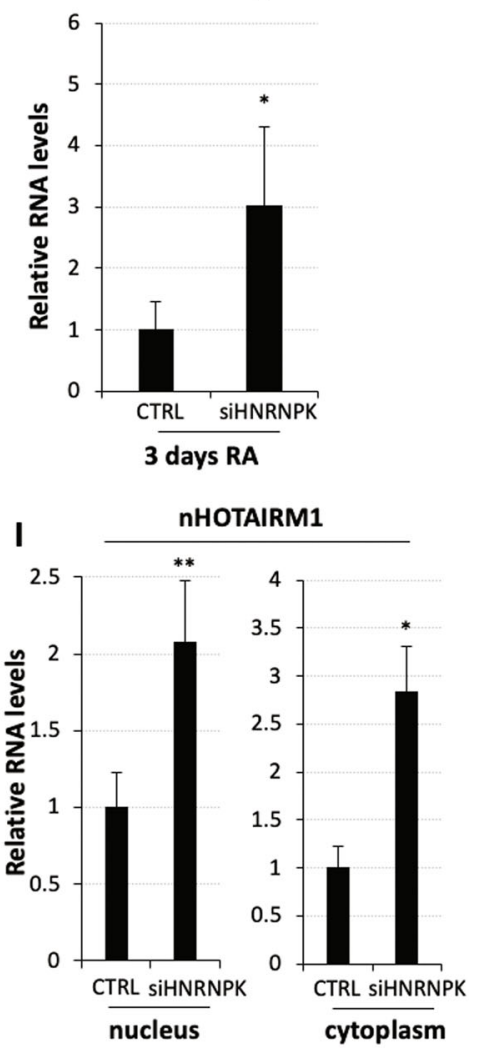

Fig. 5 HNRNPK is a regulator of nHOTAIRM1 metabolism. a qRT-PCR analysis of nHOTAIRM1 from RAP assay in differentiating MNs. RNA enrichments over Input, in nHOTAIRM1 or U1 pull-down fractions are reported. Data expressed as percentage of Input, $N=1$. b Consensus between predicted and experimentally validated interactors of nHOTAIRM1. We report a ROC (receiving operating characteristics) with AUC (area under the curve) of 0.75 between experimental and computational results. We consider as positives all the proteins with catRAPID interaction propensities $>2$ and RAP-MS NSAF scores $>0.01$. c Immunoblot analysis of HNRNPK protein upon siRNA-mediated HNRNPK knockdown in 3-day RA-treated SH-SY5Y cells. Scrambled siRNAs were used as negative control (CTRL). Relative quantity (RQ) is expressed with respect to control cells set as 1 and reported below each lane. HNRNPK levels were quantified relative to GAPDH. $N=1$. d qRT-PCR analysis of nHOTAIRM1 expression upon siRNA-mediated HNRNPK knockdown in 3-day RA-treated SH-SY5Y cells. Scrambled siRNAs were used as negative control (CTRL). Data are relative to GAPDH. N=4, ${ }^{*} P \leq 0.01$. e qRT-PCR analysis of HOTAIRM1 primary transcript (precursor) upon siRNA-mediated HNRNPK knockdown in 3-day RA-treated SH-SY5Y cells. Details as in (d). $N=3,{ }^{*} P \leq 0.05$. f qRT-PCR analysis of HOTAIRM1_2 (myeloid transcript) upon siRNA-mediated HNRNPK knockdown in 3-day RAtreated SH-SY5Y cells. Amplifications were performed through primers Ex1/3Fw and Ex3Rev2 (see Fig. 2a). Details as in (d). ${ }^{*} P \leq 0.05$. $\mathbf{g}$ qRT-PCR analyses of NEUROG2 and its downstream pathway genes (NEUROD and ASCL1) upon siRNA-mediated HNRNPK knockdown in 3-day RA-treated SHSY5Y cells. Expression peaks are set as $1 . N=2$ or 4 , depending on the target. ${ }^{*} P \leq 0.05,{ }^{*} P \leq 0.01$. $\mathbf{h}$ Immunoblot analysis of NEUROG2 protein levels upon siRNA-mediated HNRNPK knockdown in 3-day RA-treated SH-SY5Y cells. Details and statistics as in (c). $\mathbf{i}$ qRT-PCR analyses of nHOTAIRM1 in nuclear (left histogram) or cytoplasmic (right histogram) fractions from 3-day RA-treated SH-SY5Y cells, upon HNRNPK knockdown. Expression in scramble-transfected cells was set as 1 (CTRL). Normalisation was performed on total RNA. $N=4,{ }^{*} P \leq 0.05$, ${ }^{* *} P \leq 0.01$. 

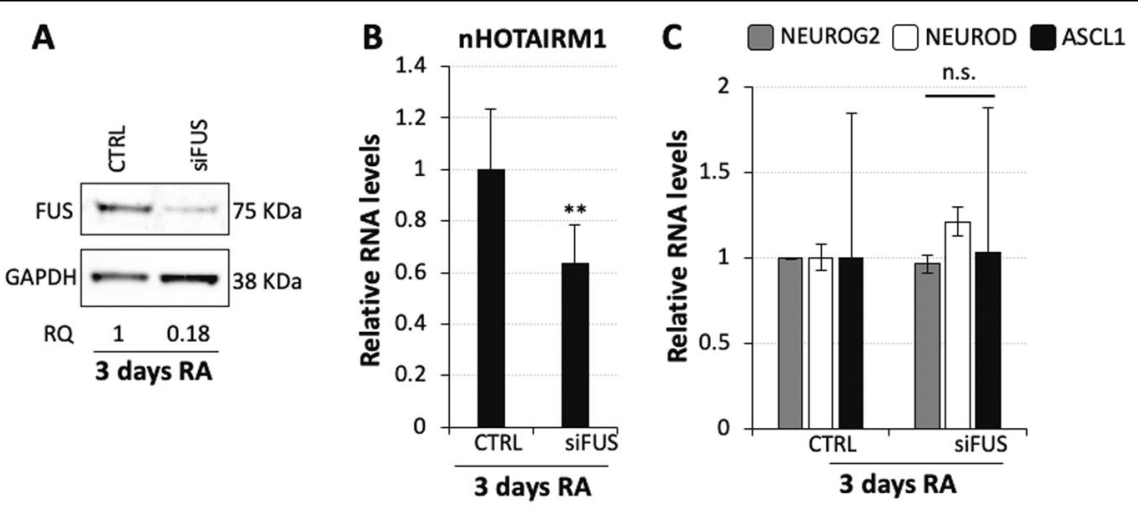

D

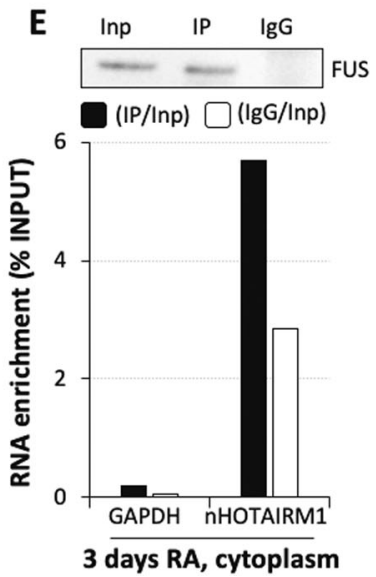

Fig. 6 FUS regulates the levels of cytoplasmic nHOTAIRM1. a Immunoblot analysis of FUS protein upon siRNA-mediated FUS knockdown in 3day RA-treated SH-SY5Y cells. Scrambled siRNAs were used as negative control (CTRL). Relative quantity (RQ) was expressed with respect to control cells set as 1 and reported below each lane. FUS levels were quantified relative to GAPDH. N=1. b qRT-PCR analysis of nHOTAIRM1 expression upon siRNA-mediated FUS knockdown in 3-day RA-treated SH-SY5Y cells. Expression in scramble-transfected cells (CTRL) is set as 1. Data are expressed relative to GAPDH. $N=3$, ${ }^{*} P \leq 0.01$. c qRT-PCR analyses of NEUROG2, NEUROD and ASCL1 upon siRNA-mediated FUS knockdown in 3-day RA-treated SH-SY5Y cells. Details as in (b). N=2 or 3, depending on the target. $\mathbf{d}$ qRT-PCR analyses of nHOTAIRM1 in nuclear (left histogram) or cytoplasmic (right histogram) fractions from RA-treated SH-SY5Y cells, upon FUS knockdown. Expression in scramble transfected cells (CTRL) was set as 1.

Normalisation was performed on total RNA. $N=2$ or 3, depending on the sample. ${ }^{* *} P \leq 0.01$. e CLIP assay for FUS in the cytoplasmic fraction of 3-day RA-treated SH-SY5Y cells. Upper panel: immunoblot analysis of FUS in Input (Inp) extract, immunoprecipitated (IP) and IgG (IgG) protein fractions. Lower panel: qRT-PCR analysis of RNA enrichment over Input, in IP and IgG fractions. Data are expressed as Input percentage. GAPDH was used as a negative control. $N=1$.

promotes neuronal differentiation ${ }^{11}$, and ASCL1, whose repression switches the cell fate from proliferation to differentiation during neural development ${ }^{41}$. This demonstrates that, by controlling NEUROG2 expression, nHOTAIRM1 drives the downstream gene cascade.

At the mechanistic level, we found that nuclear nHOTAIRM1 functions as a trans-acting molecule that mediates the epigenetic control of NEUROG2 expression by recruiting $\mathrm{PRC} 2$, which deposits the H3K27me3 repressive mark ${ }^{42,44,45}$ on NEUROG2 promoter.

The interaction between lncRNAs and PRC2 has been extensively debated. The binding of PRC2 to the RNA may be promiscuous or specific, depending on the experimental conditions and the cellular context ${ }^{65-69}$. In living cells, several parameters conferring specificitysuch as the occurrence of bridging factors-should be considered. Even though RIP and FISH assays evidenced the association of nHOTAIRM1 with PRC2 in differentiating neurons, none of these methods can indicate whether the interaction is direct or not. The catRAPID algorithm ${ }^{51,52}$ predicted that neither EZH2 nor SUZ12 have strong binding propensities to nHOTAIRM1 (scores $<1.5$, Dataset 2), suggesting that the interaction of the lncRNA with PRC2 is mediated by bridging proteins, which will be investigated in the future. Consistently, PRC2 components were not found among nHOTAIRM1 direct interactors by RAP-MS.

Being implicated in a relevant biological pathway, the levels of nHOTAIRM1 must be finely regulated. This is suggested by its expression profile, which is modulated along iPSC differentiation (Fig. 1a), and by the findings that both decrease and increase of nHOTAIRM1 


\section{NEURONAL DIFFERENTIATION}

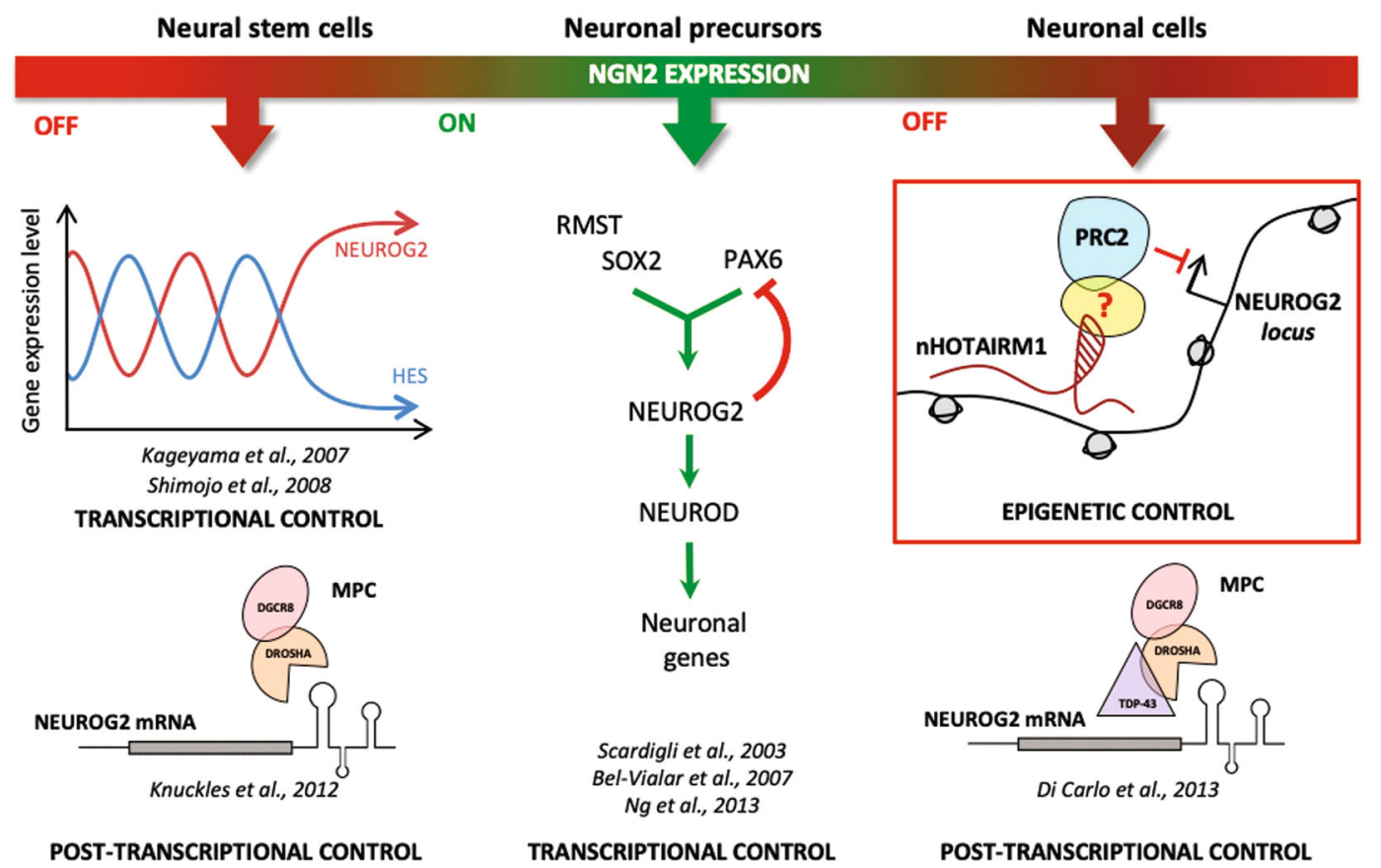

Fig. 7 A novel layer of NEUROG2 regulation. Epigenetic, transcriptional and post-transcriptional regulatory mechanisms converging on NEUROG2 contribute to its transitory expression during neuronal differentiation. The role of $\mathrm{nHOTAIRM} 1$ as a scaffold that coordinates the recruitment of the repressive epigenetic machinery PRC2 to NEUROG2 gene promoter in differentiating neurons is highlighted (red box).

abundance affect NEUROG2 cascade (Figs. 3e, g, 5d, g). This study identifies two direct protein interactors, the multifunctional RNA-binding proteins HNRNPK and FUS, contributing to the control of nHOTAIRM1 expression levels. HNRNPK may affect nHOTAIRM1 metabolism at the transcriptional and splicing levels. Splicing control is particularly relevant since it establishes the ratio between the neuronal and myeloid transcripts, which are expressed in a cell-specific manner during the differentiation processes (Fig. $2 \mathrm{~b}$ and refs. ${ }^{20,22}$ ). Therefore, HNRNPK may be included in the regulatory cascade governing neuronal differentiation (Fig. S9). Differently, the other interactor FUS cannot be integrated in NEUROG2 neurogenic pathway, since it affects the level of the cytoplasmic nHOTAIRM1 possibly conferring stability to the transcript and mediating additional functions of the lncRNA.

Besides HNRNPK and FUS, other factors were unveiled as direct nHOTAIRM1 interactors. Some of them, as HNRNP $\mathrm{H} 2$ and $\mathrm{F}$ are nuclear regulators of alternative splicing $^{70}$, whereas others, such as HNRNPA1 $1^{71}$ and the SR proteins SFPQ, SRSF1, SRSF3, SRSF7, shuttle between the nucleus and the cytoplasm ${ }^{72}$. This suggests a wide spectrum of additional activities for nHOTAIRM1, both as a splicing factor hijacker in the nucleus, as a translational regulator in the cytoplasm and as an export adaptor for the proteins that shuttle between the two compartments $^{72}$. Therefore, we cannot exclude the possibility that nHOTAIRM1 might contribute to neuronal differentiation through additional pathways, besides the NEUROG2 cascade.

In conclusion, this study allowed us to add a new tile to the mosaic of NEUROG2 regulation (Fig. 7) and revealed a novel role for the lncRNA nHOTAIRM1 in shaping the outcome of neurogenesis.

\section{Acknowledgements}

We thank M. Arceci and M. Marchioni for technical support, M. Sorci and A. Fatica for kindly providing NB4 cells and differentiation protocols, and IGBMC - Plateforme de Protéomique (Illkirch, FRANCE) for mass spectrometry analysis.

\section{Funding}

This work was partially supported by grants from Epigen-Epigenomics Flagship Project and PRIN 2017 (Prot. 2017P352Z4) to E.C., from Sapienza University (Prot. RM11715C7C8176C1 and Prot. RM11916B7A39DCE5) and FFABR Anvur (2017) to M.B., and from European Research Council (ASTRA_ 855923) to G.G.T.

\section{Author details}

'Department of Biology and Biotechnology Charles Darwin, Sapienza University of Rome, Rome, Italy. ${ }^{2}$ Center for Life Nano Science@Sapienza, Istituto Italiano di Tecnologia, Rome, Italy. ${ }^{3}$ Centre for Genomic Regulation, The Barcelona Institute for Science and Technology, Barcelona, Spain. ${ }^{4}$ Institució Catalana de Recerca i Estudis Avançats and Universitat Pompeu Fabra, Barcelona, Spain. ${ }^{5}$ Institute of Molecular Biology and Pathology, National Research Council, Rome, Italy. ${ }^{6}$ Present address: Nouscom, Rome, Italy. ${ }^{7}$ Present 
address: The BioRobotics Institute and Department of Excellence in Robotics \& Al, Scuola Superiore Sant'Anna, Pisa, Italy. ${ }^{8}$ Present address: Department of Obstetrics \& Gynecology, Stanford University, Stanford, CA, USA

\section{Author contributions}

J.R. analysed SH-SY5Y differentiation, performed HOTAIRM1 functional characterisation and HNRNPK knockdown; V.M. performed SH-SY5Y differentiation, HOTAIRM1 molecular characterisation and ChIP experiments, P.T. performed IPSC and SH-SY5Y differentiation, CLIP and RAP-MS assays and FUS knockdown; T.S. performed and analysed imaging experiments; A.A. applied catRAPID calculations; M.G.G. cultured IPSCs and performed differentiations; F.I. contributed to IPSC and SH-SY5Y differentiation analyses; P.L. performed RIP assays and coordinated the experimental activity; A.C. setup and supervised the RAP-MS experiment; G.G.T. supervised bioinformatics analyses; A.R. supervised the iPSC manipulations; M.B. supervised and interpreted ChIP experiments and revised the paper; J.R. and P.T. performed statistical analyses; E.C. and P.L. conceived the project, interpreted the results and wrote the paper. E.C. secured the funding.

\section{Conflict of interest}

The authors declare that they have no conflict of interest.

\section{Publisher's note}

Springer Nature remains neutral with regard to jurisdictional claims in published maps and institutional affiliations.

Supplementary Information accompanies this paper at (https://doi.org/ 10.1038/s41419-020-02738-w).

Received: 29 December 2019 Revised: 15 June 2020 Accepted: 18 June 2020

Published online: 13 July 2020

\section{References}

1. Mayer-Proschel, M., Kalyani, A. J., Mujtaba, T. \& Rao, M. S. Isolation of lineagerestricted neuronal precursors from multipotent neuroepithelial stem cells. Neuron 19, 773-785 (1997).

2. Bertrand, N., Castro, D. S. \& Guillemot, F. Proneural genes and the specification of neural cell types. Nat Rev. Neurosci. 3, 517-530 (2002).

3. Liu, Y. \& Zhang, Y. ETV5 is essential for neuronal differentiation of human neural progenitor cells by repressing NEUROG2 expression. Stem Cell Rev. Rep. 15. 703-716 (2019).

4. Shimojo, H., Ohtsuka, T. \& Kageyama, R. Oscillations in notch signaling regulate maintenance of neural progenitors. Neuron 58, 52-64 (2008).

5. Kageyama, R., Ohtsuka, T. \& Kobayashi, T. The Hes gene family: repressors and oscillators that orchestrate embryogenesis. Development 134, 1243-1251 (2007).

6. Ma, Q., Kintner, C. \& Anderson, D. J. Identification of neurogenin, a vertebrate neuronal determination gene. Cell 87, 43-52 (1996).

7. Nieto, M., Schuurmans, S., Britz, O. \& Guillemot, F. Neural bHLH genes control the neuronal versus glial fate decision in cortical progenitors. Neuron $\mathbf{2 9}$ 401-413 (2001).

8. Sun, Y. et al. Neurogenin promotes neurogenesis and inhibits glial differentiation by independent mechanisms. Cell 104, 365-376 (2001).

9. Scardigli, R., Baumer, N., Gruss, P., Guillemot, F. \& Le Roux, I. Direct and concentration-dependent regulation of the proneural gene Neurogenin2 by Pax6. Development 130, 3269-3281 (2003).

10. Ng, S. Y., Bogu, G. K., Soh, B. S. \& Stanton, L. W. The long noncoding RNA RMST interacts with SOX2 to regulate neurogenesis. Mol. Cell 51, 349-359 (2013).

11. Seo, S., Lim, J. W., Yellajoshyula, D., Chang, L. W. \& Kroll, K. L. Neurogenin and NeuroD direct transcriptional targets and their regulatory enhancers. EMBO J. 26, 5093-5108 (2007).

12. Bel-Vialar, S., Medevielle, F. \& Pituello, F. The on/off of Pax6 controls the tempo of neuronal differentiation in the developing spinal cord. Dev. Biol. 305 659-673 (2007).

13. Di Carlo, V. et al. TDP-43 regulates the microprocessor complex activity during in vitro neuronal differentiation. Mol. Neurobiol. 48, 952-963 (2013).
14. Briggs, J. A., Wolvetang, E. J., Mattick, J. S., Rinn, J. L. \& Barry, G. Mechanisms of long non-coding RNAs in mammalian nervous system development, plasticity, disease, and evolution. Neuron 88, 861-877 (2015).

15. Engreitz, J. M., Ollikainen, N. \& Guttman, M. Long non-coding RNAs: spatial amplifiers that control nuclear structure and gene expression. Nat. Rev. Mol. Cell Biol. 17, 756-770 (2016)

16. Laneve, P. et al. The long noncoding RNA linc-NeD125 controls the expression of medulloblastoma driver genes by microRNA sponge activity. Oncotarget $\mathbf{8}$, 31003-31015 (2017).

17. Knauss, J. L. \& Sun, T. Regulatory mechanisms of long noncoding RNAs in vertebrate central nervous system development and function. Neuroscience 235, 200-214 (2013).

18. Quan, Z., Zheng, D. \& Qing, H. Regulatory roles of long non-coding RNAs in the central nervous system and associated neurodegenerative diseases. Front. Cell Neurosci. 11, 175 (2017).

19. Bevilacqua, V. et al. Identification of linc-NeD125, a novel long non coding RNA that hosts miR-125b-1 and negatively controls proliferation of human neuroblastoma cells. RNA Biol. 12, 1323-1337 (2015).

20. Zhang, X. et al. A myelopoiesis-associated regulatory intergenic noncoding RNA transcript within the human HOXA cluster. Blood 113, 2526-2534 (2009).

21. Wang, X. Q. D. \& Dostie, J. Reciprocal regulation of chromatin state and architecture by HOTAIRM1 contributes to temporal collinear HOXA gene activation. Nucleic Acids Res. 45, 1091-1104 (2017).

22. Zhang, X., Weissman, S. M. \& Newburger, P. E. Long intergenic non-coding RNA HOTAIRM1 regulates cell cycle progression during myeloid maturation in NB4 human promyelocytic leukemia cells. RNA Biol. 11, 777-787 (2014).

23. Lenzi, J. et al. ALS mutant FUS proteins are recruited into stress granules in induced pluripotent stem cells (iPSCs) derived motoneurons. Dis. Model. Mech. 8, 755-766 (2015)

24. Ballarino, M. et al. Deficiency in the nuclear long noncoding RNA Charme causes myogenic defects and heart remodeling in mice. EMBO J. 37, e99697 (2018).

25. McHugh, C. A. \& Guttman, M. RAP-MS: a method to identify proteins that interact directly with a specific RNA molecule in cells. Methods Mol. Biol. 1649 473-488 (2018).

26. Bellucci, M., Agostini, F., Masin, M. \& Tartaglia, G. G. Predicting protein associations with long noncoding RNAs. Nat. Methods 8, 444-445 (2011).

27. Marchese, D. et al. Discovering the $3^{\prime}$ UTR-mediated regulation of alphasynuclein. Nucleic Acids Res. 45, 12888-12903 (2017)

28. Cid-Samper, F. et al. An integrative study of protein-RNA Condensates Identifies Scaffolding RNAs and Reveals Players in Fragile X-Associated Tremor/ Ataxia Syndrome. Cell Rep. 25, 3422-3434 (2018).

29. Lang, B., Armaos, A. \& Tartaglia, G. G. RNAct: protein-RNA interaction predictions for model organisms with supporting experimental data. Nucleic Acids Res. 47, D601-D606 (2019).

30. Lin, M. et al. RNA-Seq of human neurons derived from iPS cells reveals candidate long non-coding RNAs involved in neurogenesis and neuropsychiatric disorders. PLoS ONE 6, e23356 (2011).

31. De Santis, R. et al. FUS mutant human motoneurons display altered transcriptome and microRNA pathways with implications for ALS pathogenesis. Stem Cell Rep. 9, 1450-1462 (2017).

32. Pezzini, F. et al. Transcriptomic profiling discloses molecular and cellular events related to neuronal differentiation in SH-SY5Y neuroblastoma cells. Cell. Mol. Neurobiol. 37, 665-682 (2017).

33. Shipley, M. M. Mangold, C. A. \& Szpara, M. L. Differentiation of the SH-SY5Y human neuroblastoma cell line. J. Vis. Exp. 108, 53193 (2016).

34. Gimenez-Cassina, A., Lim, F. \& Diaz-Nido, J. Differentiation of a human neuroblastoma into neuron-like cells increases their susceptibility to transduction by herpesviral vectors. J. Neurosci. Res. 84, 755-767 (2006).

35. Kovalevich, J. \& Langford, D. Considerations for the use of SH-SY5Y neuroblastoma cells in neurobiology. Methods Mol. Biol. 1078, 9-21 (2013).

36. Cheung, Y. T. et al. Effects of all-trans-retinoic acid on human SH-SY5Y neuroblastoma as in vitro model in neurotoxicity research. Neurotoxicology $\mathbf{3 0}$ 127-135 (2009).

37. Di Rocco, G. et al. Interplay of the E box, the Cyclic AMP Response Element and HTF4/HEB in transcriptional regulation of the neurospecific, neurotrophininducible vgf gene. Mol. Cell. Biol. 17, 1244-1253 (1997).

38. Constantinescu, R., Constantinescu, A. T., Reichmann, H. \& Janetzky, B. Neuronal differentiation and long-term culture of the human neuroblastoma line SH-SY5Y. J. Neural Transm. Suppl. 72, 17-28 (2007).

39. Schwab, M. MYCN in neuronal tumours. Cancer Lett. 204, 179-187 (2004). 
40. Lennox, K. A. \& Behlke, M. A. Mini-review on current strategies to knockdown long non-coding RNAs. J. Rare Dis. Res. Treat. 1, 66-70 (2016).

41. Gustincich, S., Zucchelli, S. \& Mallamaci, A. The Yin and Yang of nucleic acidbased therapy in the brain. Prog. Neurobiol. 155, 194-211 (2017).

42. Castro, D. S. et al. A novel function of the proneural factor Ascl1 in progenitor proliferation identified by genome-wide characterization of its targets. Genes Dev. 25, 930-945 (2011).

43. Cao, R. et al. Role of histone $\mathrm{H3}$ lysine 27 methylation in Polycomb-group silencing. Science 298, 1039-1043 (2002).

44. Hansen, K. H. et al. A model for transmission of the H3K27me3 epigenetic mark. Nat. Cell Biol. 10, 1291-1300 (2008).

45. Schuettengruber, B., Chourrout, D., Vervoort, M., Leblanc, B. \& Cavalli, G. Genome regulation by polycomb and trithorax proteins. Cell 128, 735-745 (2007).

46. Pengelly, A. R., Copur, Ö., Jäckle, H., Herzig, A. \& Müller, J. A histone mutant reproduces the phenotype caused by loss of histone-modifying factor Polycomb. Science 339, 698-699 (2013).

47. McHugh, C. A., Russell, P. \& Guttman, M. Methods for comprehensive experimental identification of RNA-protein interactions. Genome Biol. 15, 203 (2014).

48. De Santis, R. et al. Direct conversion of human pluripotent stem cells into cranial motor neurons using a piggyBac vector. Stem Cell Res. 29, 189-196 (2018).

49. Garone, M.G. et al. Conversion of human induced pluripotent stem cells (iPSCS) into functional spinal and cranial motor neurons using PiggyBac vectors. J. Vis. Exp. 147, e59321. https://doi.org/10.3791/59321 (2019).

50. McHugh, C. A. et al. The Xist IncRNA interacts directly with SHARP to silence transcription through HDAC3. Nature 521, 232-236 (2015).

51. Agostini, F. et al. catRAPID omics: a web server for large-scale prediction of protein-RNA interactions. Bioinformatics 29, 2928-2930 (2013).

52. Cirillo, D. et al. Quantitative predictions of protein interactions with long noncoding RNAs. Nat. Methods 14, 5-6 (2016).

53. Thomas-Jinu, S. et al. Non-nuclear pool of splicing factor SFPQ regulates axonal transcripts required for normal motor development. Neuron 94 322-336 (2017)

54. Jeong, S. S. R. Proteins: binders, regulators, and connectors of RNA. Mol. Cells 40, 1-9 (2017).

55. Geuens, T., Bouhy, D. \& Timmerman, V. The hnRNP family: insights into their role in health and disease. Hum. Genet. 135, 851-867 (2016).

56. Lagier-Tourenne, C., Polymenidou, M. \& Cleveland, D. W. TDP-43 and FUS/TLS: emerging roles in RNA processing and neurodegeneration. Hum. Mol. Genet. 19, 46-64 (2010).
57. Li, J. et al. Identification of human neuronal protein complexes reveals biochemical activities and convergent mechanisms of action in autism spectrum disorders. Cell Syst. 1, 361-374 (2015).

58. $\mathrm{Xu}, \mathrm{Y}$. et al. New Insights into the interplay between non-coding RNAs and RNA-binding protein HnRNPK in regulating cellular functions. Cells 8, 62 (2019).

59. Svetoni, F. et al. Post-transcriptional regulation of FUS and EWS protein expression by miR-141 during neural differentiation. Hum. Mol. Genet. 26 2732-2746 (2017)

60. Kwiatkowski, T. J. Jr et al. Mutations in the FUS/TLS gene on chromosome 16 cause familial amyotrophic lateral sclerosis. Science $\mathbf{3 2 3}$ 1205-1208 (2009)

61. Vance, $\mathrm{C}$. et al. Mutations in FUS, an RNA processing protein, cause familial amyotrophic lateral sclerosis type 6. Science 323, 1208-1211 (2009).

62. Capauto, D. et al. A regulatory circuitry between Gria2, miR-409, and miR-495 is affected by ALS FUS mutation in ESC-derived motor neurons. Mol. Neurobiol. 55, 7635-7651 (2018).

63. Yuan, L. \& Hassan, B. A. Neurogenins in brain development and disease: an overview. Arch. Biochem. Biophys 558, 10-13 (2014).

64. Knuckles, P. et al. Drosha regulates neurogenesis by controlling neu rogenin 2 expression independent of microRNAs. Nat. Neurosci. 15 962-969 (2012).

65. Davidovich, C., Zheng, L., Goodrich, K. J. \& Cech, T. R. Promiscuous RNA binding by Polycomb repressive complex 2. Nat. Struct. Mol. Biol. 20, 1250-1257 (2013)

66. Kaneko, S., Son, J., Shen, S. S., Reinberg, D. \& Bonasio, R. PRC2 binds active promoters and contacts nascent RNAs in embryonic stem cells. Nat. Struct. Mol. Biol. 20, 1258-1264 (2013).

67. Beltran, M. et al. The interaction of PRC2 with RNA or chromatin is mutually antagonistic. Genome Res. 26, 896-907 (2016).

68. Davidovich, $\mathrm{C}$. et al. Toward a consensus on the binding specificity and promiscuity of PRC2 for RNA. Mol. Cell 57, 552-558 (2015).

69. Cipriano, A. \& Ballarino, M. The ever-evolving concept of the gene: the use of RNA/protein experimental techniques to understand genome functions. Front. Mol. Biosci. 5, 20 (2018).

70. Gautrey, H. et al. SRSF3 and hnRNP H1 regulate a splicing hotspot of HER2 in breast cancer cells. RNA Biol. 12, 1139-1151 (2015).

71. Jean-Philippe, J., Paz, S. \& Caputi, M. hnRNP A1: the Swiss army knife of gene expression. Int. J. Mol. Sci. 14, 18999-19024 (2013).

72. Cáceres, J. F., Screaton, G. R. \& Krainer, A. R. A specific subset of SR proteins shuttles continuously between the nucleus and the cytoplasm. Genes Dev. 12 55-66 (1998). 